\title{
Comparison of Various Spectral Models for the Prediction of the 100-Year Design Wave Height
}

\author{
Sayyid Zainal Abidin Syed Ahmad ${ }^{1,2, *}$, Mohd Khairi Abu Husain ${ }^{1}$, Noor Irza Mohd Zaki ${ }^{1}$, \\ Mohd Hairil Mohd ${ }^{2}$ and Gholamhossein Najafian ${ }^{3}$ \\ ${ }^{1}$ Universiti Teknologi Malaysia, 54100 Kuala Lumpur, Malaysia \\ ${ }^{2}$ Universiti Malaysia Terengganu, 21030 Kuala Nerus, Malaysia \\ ${ }^{3}$ School of Engineering, Universiti of Liverpool, Liverpool, United Kingdom
}

\begin{abstract}
Offshore structures are exposed to random wave loading in the ocean environment, and hence the probability distribution of the extreme values of their response to wave loading is required for their safe and economical design. In most cases, the dominant load on offshore structures is due to wind-generated random waves where the ocean surface elevation is defined using appropriate ocean wave energy spectra. Several spectral models have been proposed to describe a particular sea state that is used in the design of offshore structures. These models are derived from analysis of observed ocean waves and are thus empirical in nature. The spectral models popular in the offshore industry include Pierson-Moskowitz spectrum and JONSWAP spectrum. While the offshore industry recognizes that different methods of simulating ocean surface elevation lead to different estimation of design wave height, no systematic investigation has been conducted. Hence, the aim of this study is to investigate the effects of predicting the 100-year responses from various wave spectrum models. In this paper, the Monte Carlo time simulation (MCTS) procedure has been used to compare the magnitude of the 100-year extreme responses derived from different spectral models. Additionally, the linear random wave theory (LRWT) was implemented to simulate the offshore structural responses due to random wave loading. The models have been tested for three different environmental conditions represented by $\mathrm{Hs}=15 \mathrm{~m}, 10 \mathrm{~m}$ and $5 \mathrm{~m}$ respectively. The accuracy of the predictions of the 100 -year responses from Pierson-Moskowitz and JONSWAP spectrums will then be investigated.
\end{abstract}

\section{Introduction}

The study of oceanography has always featured irregular waves. It became a non-uniform series of waves, inconstant of height and length, continuing in unsteady and unreliable sequences. In reality, there has never been seen a constant progression of identical waves at sea. The term random phenomenon [1] is closely associated with the continuous change in wave height, length and breadth dimension as well. Instead, the surface elevation or sea

\footnotetext{
* Corresponding author: s.zainal@umt.edu.my
} 
surface, also known as gravity waves, is composed of waves of fluctuating heights and periods moving in distinct directions. Wind waves are highly irregular concerning their direction, amplitude and frequency, and irregular random nature. Therefore, the shape of surface elevation in the existence of wind waves cannot be deterministically described.

Recognising the fundamental randomness of the surface elevation is the initial step before analysis. It becomes necessary to view the characteristics of the sea surface in proper ways. There are two ways to represent the irregular nature of wind waves in upsurges. First, the usage of the wave energy spectrum and second, use of the statistical probability distributions of single wave characteristics. Larsén et al. [2] studied how a statistical methodology for the estimation of extreme waves was predicted. Spectra description of the sea states is an essential input in the study of wave characteristics even in marine structures and vessels. The spectrum measured in a particular point of the ocean is generally considered as the sum of wave classifications (parameters) produced by an event separated either in space, in time, or both. In general, the spectrum of the sea surface does not follow any specific mathematical form. However, under certain wind conditions, the spectrum does have a specific shape. A series of empirical expressions have been developed, which can be fitted to the spectrum of the sea surface elevation. These are called parametric spectrum models. The modified P-M model for "Fully Developed Sea" and JONSWAP model for fetch limited "Developing Sea" are the widely accepted and used models all around the world for decades. In this paper, the linear wave theory will be referred to as Linear Random Wave Theory (LRWT) or Airy Wave Theory [3].

According to Mihram [4], a discussion of the elements of queues reveals an exemplary class of complex systems whose simulation often is best accomplished by the application of the Monte Carlo method and then Jensen [5] studied the predictions of extreme value on increasing load spectrum by using Monte Carlo approach. Monte Carlo simulation is a computerized mathematical technique that allows people to account for risk in quantitative analysis and decision making [6]. Monte Carlo simulation performs risk analysis by building models of possible results by substituting a range of values that come up with a probability distribution which is any factor that has inherent uncertainty. It then calculates results over and over, each time using a different set of random values from the probability functions. Depending upon the number of uncertainties and the ranges specified for them, a Monte Carlo simulation could involve thousands or tens of thousands of recalculations before it is complete. Monte Carlo simulation produces distributions of possible outcome values. Abu Husain et al. [7] had applied Monte Carlo for predicting the short-term and long-term statistical properties of the response of an offshore structure to random wave loading in addition to, obtaining the estimates of extreme waves which is the standard approach that needs to fit the data to an extreme probability distribution (Weibull, Gumbel, Generalized Pareto etc.). By extrapolation, extreme waves with a low probability of occurrence (e.g. a wave that will only occur one time in a period of 100 years) can be estimated [8]. These results are used as design conditions for offshore structures.

\section{Wave theory}

Waves can be modelled by using linear and non-linear terms. In this paper, the linear wave theory will be referred to as Airy Wave Theory or Linear Random Wave Theory (LRWT). Airy wave theory was introduced by Airy in 1842 [9]. It was a relatively simple theory of wave motion. The differential equations of water particle behaviour is a linear solution concept to this theory. 


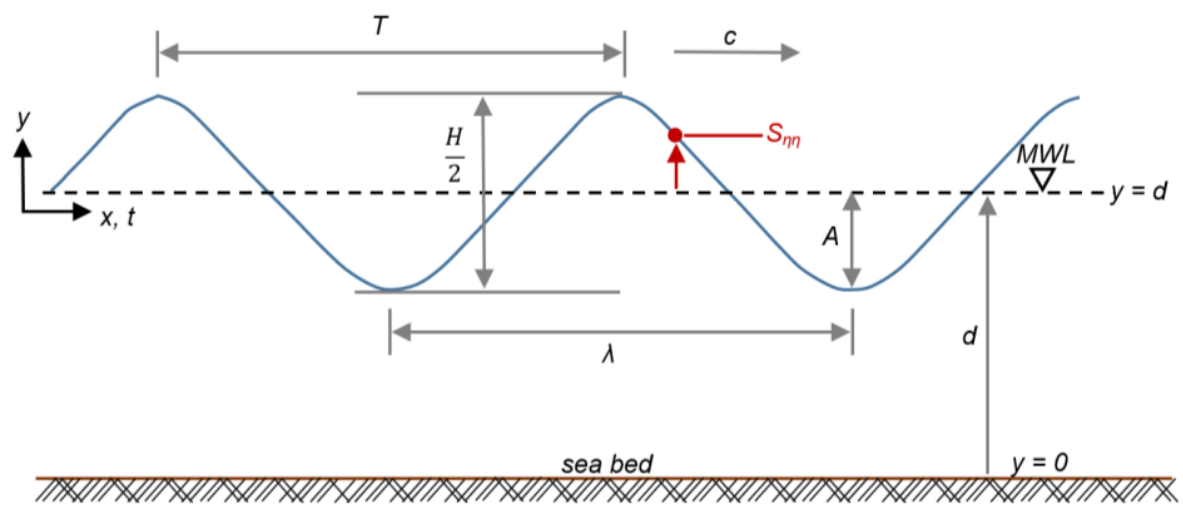

Fig. 1. Wave arrangements

The description presumes a sinusoidal wave theory where A is amplitude, Snn is the surface elevation frequency spectrum, $c$ is celerity, $\lambda$ is wavelength, $T$ is wave period, $d$ is the water depth, $\mathrm{x}$ is horizontal direction for distance, $\mathrm{t}$ is horizontal direction for time, $\mathrm{z}$ is vertical direction for wave height and MWL is mean water level (water sea surface). This theory is valuable for preliminary calculations and for revealing the basic characteristics of wave-induced water motion [10]. On the other hand, the use of simple wave theories is common in engineering solutions in Fig. 1. The simple wave theories produce good mathematical models because the long crest periodic waves are one the components of an irregular sea [11]. There are two main categories of waves; the regular waves and irregular waves.

\subsection{Regular and irregular}

Simple waves can be defined in simple mathematical terms, which allow for a better understanding of the motion and behaviour of waves. There are two simple waves; sinusoidal or monochromatic waves. A single sine or cosine function is able to depict their surface profile. This can be measured and analysed based on their basic characteristics remaining constant. Once the wavelength, height, period and depth are known, for this reason, their motion and behaviour can be fully described. On the other hand, simple waves are rarely seen in nature and are more successfully generated in a laboratory [12].

Regular waves (wave component) has a single frequency (wavelength) and amplitude (height).Irregular waves can be viewed as the superposition of a number of regular waves (wave components) with different frequencies and amplitudes. Regarding to irregular waves imply that all the wave components are in the same direction. It can also be called uni-directional irregular waves, known as long-crested irregular waves meanwhile irregular wave components are often multi-directional and this direction of an irregular wave is known as short-crested waves. In nature, wind waves are very complex. Looking out at the sea, one never sees a constant progression of identical waves. Instead, the sea surface is composed of waves of varying heights and periods moving in differing directions. When the wind blows and the waves grow in response, the seas tend to be confused: a wide range of heights and periods is observed. Therefore with their perfect regularity, simple waves do not accurately depict the random nature of ocean waves [13].

\subsection{Random waves}

It becomes necessary to treat the characteristics of the sea surface in statistical and spectra terms once the fundamental randomness of the sea surface is recognized. The combination 
of many wave components has a significant affect on ocean surface. Winds in different regions of the ocean may generate these individual components and form complex waves that are propagating to the point of observation. This would be seen when a recorder was to measure waves at a fixed location of the ocean, record of the wave surface would be rather irregular and random, a non-repeating wave profile. There is significant variability in height and period from wave to wave, although individual waves can be identified. Consequently, a statistical or probabilistic is simply a technique to indicate the severity of wave conditions in order to explain the wave characteristics such as height, period, etc. [14].

The harmonic analysis (Fourier analysis) is a strong tool used to describe a random surface with sinusoidal components. Rough ocean surface can be represented by a mathematical expression in Linear Random Wave Theory formulation. This representation describes the harmonic analysis of wind-driven sea surface waves with some simplifications. This situation considers all surface wave components with wave amplitude, wave frequency and phase angle coming from various directions as a single wave component [15].

\subsection{Theoretical wave spectra}

In wave spectra, there are two main approaches for treating complex waves: spectra analysis and wave-by-wave (wave train) analysis. The more powerful and popular of these two approaches is the spectra analysis. The spectra modelling of the sea states is the basic description of the probabilistic nature of the sea surface or surface elevation. It is based on the assumption that the sea surface elevation can be modelled as an ergodic, and thus stationary, Gaussian stochastic process [16].

Mathematically, spectra analysis is based on the Fourier Transform of the sea surface. According to [17], the Fourier Transform allows any continuous, zero-mean signal - like time-series record of the sea surface elevation - to be transformed into a summation of simple sine waves. There are many forms of wave energy spectra used in practice, which are based on one or more parameters such as wind speed, significant wave height, wave period, shape factors and so on that depend on what spectrum to be used. In this study, two theoretical spectra are selected. Each has been explained in few details.

\subsubsection{Pierson-Moskowitz Spectrum (P-M Spectrum)}

Initially, P-M spectrum described one-parameter only based on the wind speed (Uw) for fully developed seas [18]. Nonetheless, most sea states cannot be achieved for fully developed seas. The reason is due to the different wind speed and direction, the fetch too short or the wind duration is not long enough when encountering strong winds and high waves. Then, the presence two-parameter spectrum would be better used for the significant wave height and mean zero up-crossing period parameters.

Subsequently, eliminating the wind speed from the one-parameter P-M spectrum by incorporating Eq. (1), an improved P-M frequency spectrum [19] describes the waves developed with no limitations in fetch and wind duration which are called fully developed. In this study, the following definition of the P-M spectrum can be used in the significant wave height (Hs) and mean zero up-crossing period (Tz) as the input into two-parameter and the Pierson-Moskowitz spectrum can be written:

$$
S_{n n}(f)_{P M}=\frac{H_{g}^{2}}{4 \pi T_{z}^{4} f^{5}} \exp \left(\frac{1}{\pi T_{z}^{4} f^{4}}\right)
$$

where $S_{m n}$ is the surface elevation frequency spectrum, $\pi$ is the mathematical constant of 3.142 , $\mathrm{f}$ is the wave frequency, $\mathrm{Hs}$ is the significant wave height and $\mathrm{Tz}$ is mean zero upcrossing period. 


\subsubsection{JONSWAP spectrum}

This spectrum is an extension of the P-M spectrum which is the JONSWAP (Joint North Sea Wave Project) spectrum [20]; this is a five-parameter (fp, $\alpha, \gamma$, $\sigma \mathrm{a}$ and $\sigma \mathrm{b}$ ) or (Hs, Tp, $\gamma$, $\sigma \mathrm{a}$ and $\sigma \mathrm{b})$, where three of these parameters $(\gamma, \sigma \mathrm{a}$ and $\sigma \mathrm{b})$ are usually held constant. The JONSWAP spectrum is valid for not fully developed sea states. However, it is also used to represent fully developed sea states in which are included fetch limited seas in Eq. (2). The JONSWAP spectrum is given in the form:

$$
S_{n n}(f)_{J}=S_{n n}(f)_{P M} \gamma_{p} \exp \left[-\frac{\left(f-f_{p}\right)^{2}}{2 \sigma^{2} f_{p}^{2}}\right]
$$

The peak enhancement factor, $\gamma=1-7$ (average 3.3), is introduced to represent fetch limited wind sea affected by a parameter, $\sigma$ (the width of the spectra peak) as below in Eq. (3) and given by:

$$
\begin{aligned}
& \sigma_{a}=0.07, f \leq f_{p} \\
& \sigma_{b}=0.09, f>f_{p}
\end{aligned}
$$

where $\gamma_{p}$ is the peak enhancement factor, $f$ is the wave frequency, $f_{p}$ is the peak wave frequency, $\sigma(f)=\sigma_{\alpha}$ if $0.07, f \leq f_{p}$ "standard deviation" of the peak enhancement factor to the left and $\sigma(f)=\sigma_{b}$ if $0.09, f>f_{p}$ "standard deviation" of the peak enhancement factor to the right $S_{n n}(f)_{P M}$ is the surface elevation of P-M frequency spectrum.

\subsection{Derivation of surface elevation by linear random wave theory}

According to LRWT, unidirectional seas can be modelled as the sum of a large number of linear progressive wavelets, all travelling in the same direction with random phase angles $[15,21]$. The surface elevation at point- $x$ and time- $t$ (two independent variables) that has been used is sketched in Fig. 1:

$$
\eta(x, t)=\sum_{i=1}^{N W} A_{i} \cos \left(2 \pi f_{i} t-k_{i} x-\varphi_{i}\right)
$$

where $\eta$ is the water surface elevation, $N W$ is the total wavelet number used, $x$ is displacements, $t$ is a time, $f_{i}$ is a set of wave frequency, $\varphi_{i}$ is a random phase angle in range 0 to $2 \pi, k$ is the wave number and $A_{i}$ is the wave amplitudes determined using the nondeterministic spectra amplitude (NSA) method.

$$
A=\sqrt{2 S_{\omega}\left(f_{i}\right) \Delta f} * \sqrt{\frac{g_{i}^{2}+h_{i}^{2}}{2}}
$$

where $S_{\omega}$ is a one-sided surface elevation frequency spectrum, $\Delta f$ is the frequency interval, $g_{i}$ and $h_{i}$ are two independent and standardised Gaussian random variables.

Once the extreme surface elevation values have been obtained by repeating the previous procedure, the following plotting position equation in the Gumbel distribution is used, to estimate the probability distribution value for each ranked extreme value.

$$
\begin{array}{r}
\operatorname{Prob}\left(r_{\max }<q_{n}\right)=P_{T_{\max }}\left(q_{n}\right) \approx \frac{n-0.44}{N+0.12} \\
n=1,2,3, . ., N
\end{array}
$$

Therefore, the magnitude of the 100-year extreme surface can be calculated by the following equations: 


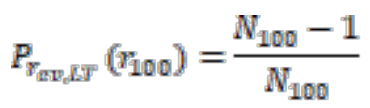

where $P_{V_{G V}, E F}$ is the long-term distribution of extreme response, $r_{100}$ is 100 -year probability distribution of extreme surface elevation and $N_{100}$ is the number of 3.64 hours during 100year.

\section{Research methodology}

In this study, probabilistic method also known as the non-deterministic method will be used to evaluate the results. It is a statistical analysis tool that estimate and representation of a randomness phenomenon. The stochastic model includes both a deterministic component and a random error component [22]. Monte Carlo is the best technique in a stochastic method for predicting responses of an offshore structure random wave [5, 6]. As shown in Fig. 2, the study process followed and orderly sequence and provided a logical framework.

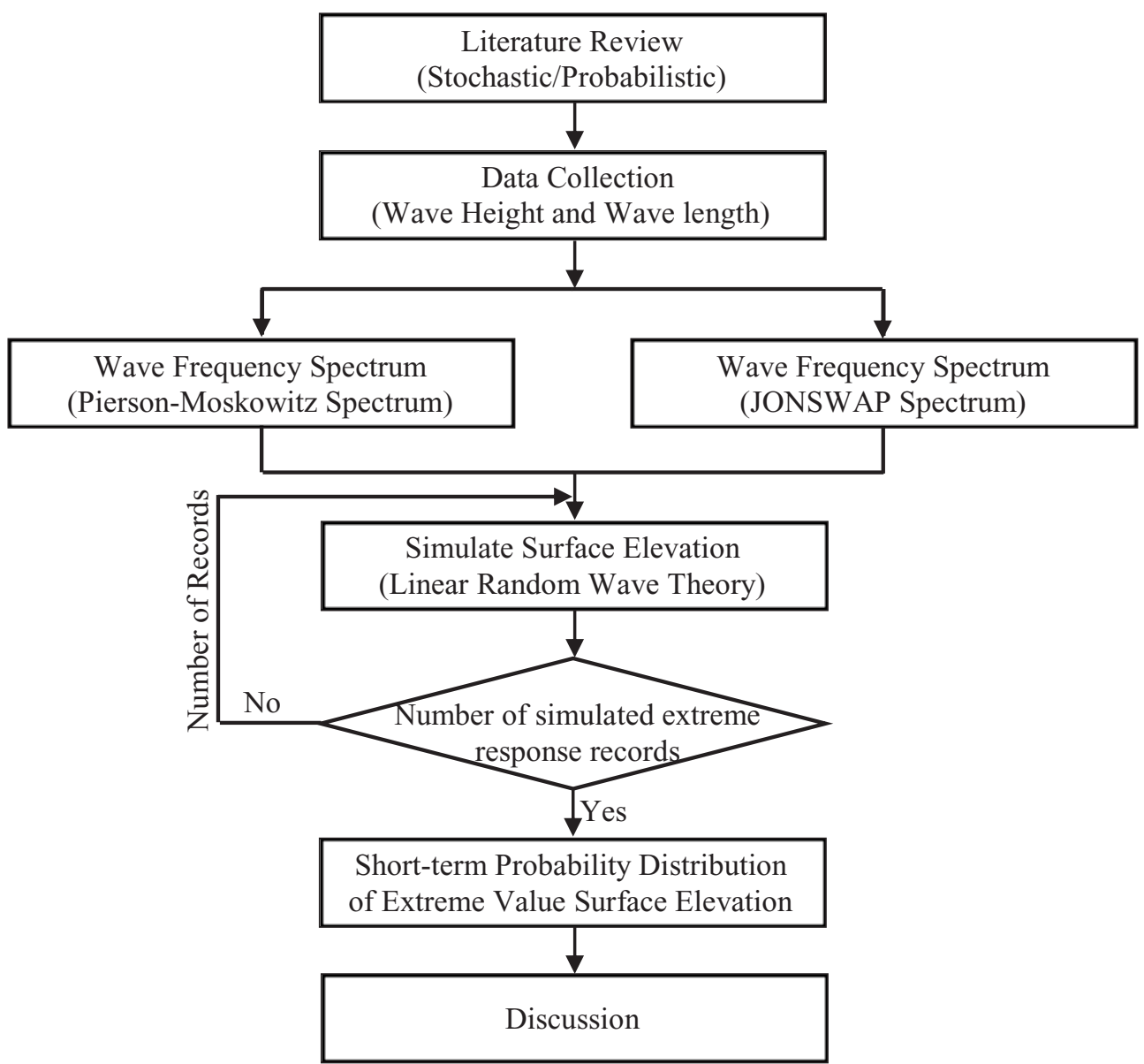

Fig. 2. Study procedure operational framework for the evaluation of short-term probability distribution of response extreme values.

The short-term analysis, will consider several sea state conditions. There are three wave heights $\left(H_{s}=5 \mathrm{~m}, 10 \mathrm{~m}, 15 \mathrm{~m}\right)$ and wave zero up-crossing periods $\left(T_{z}=7.94 \mathrm{sec}, 11.23 \mathrm{sec}\right.$, 
$13.75 \mathrm{sec}$ ) are selected. In this study, there are two wave spectra that will be examined; the Pierson-Moskowitz spectrum and JONSWAP spectrum. By using the formula given, each spectrum will produce different energy in Eq. (5). Then, surface elevation is obtained through a simulation of Linear Random Wave Theory. In calculating the surface elevation the Fourier analysis approach is used. According to Haver [23], the acceptable length of wave simulation is about 3 hours. Therefore, this study will also be run by using the Fast Fourier Transform (FFT) performed every 3.64 hours. This provides information on what frequency components represent a function that needs to perform an analysis with MATLAB will be considered in this study. Next, a sample wave record can be simulated by calculating each function in terms of sinusoidal components separately and then summation that produces spectra terms in Eq. (4). However, this procedure has a limitation on large harmonic numbers. In order to speed up the simulation, the Fast Fourier Transform (FFT) technique is much more efficient to use because the transformation of the Discrete Fourier Transform (DFT) of the surface elevation is record directly into a time $(t)$ record independent variable only. In other words, the wave simulation of surface elevation has coupled variable $x$ and $t$ into variable $t$ only through Fourier transform to become first order terms.

Lastly, the Monte Carlo simulations are performed on the available scatter data. As many as 100,000 simulated extremes of surface elevation has been simulated to verify the results from the short-term response analysis for 3 hours. Wang et al. [24] used the Gumbel distributions for modelling of extreme responses by comparing them with empirical distributions derived from extensive Monte Carlo time simulations. In this study, the Gumbel distribution would be also considered to predict an accurate estimate for 100-year the extreme value of surface elevation. Rank of all the simulated extreme values from the smallest to largest can be calculated based on Eq. (6). Gumbel distribution is a useful tool that provides to the researcher constitutes several different wave statistics in systematic ways.

\section{Results and discussion}

As previously mentioned, the aim of this study is to investigate the effects of predicting the 100year responses from various wave spectrum models. The comparison is carried out using different level of significant wave heights, $H_{S}(5 \mathrm{~m}, 10 \mathrm{~m}$, and $15 \mathrm{~m})$ values for two different ocean wave spectra (Pierson-Moskowitz and JONSWAP spectrums). The analysis is made on a simulated random wave generated using linear random wave theory (LRWT). Then, the Monte Carlo time simulation method is utilised for predicting the short-term statistical properties of the 100-year design wave height due to its capability of accounting for various nonlinearities. In this section, a comparison between two different spectra will be analysed first followed by the design wave height investigation.

\subsection{Comparison between P-M and JONSWAP spectrum}

The wave spectra can be developed by using a formula of frequency spectrum as mentioned in Eq. (1) and (2). In Fig. 3(a) can be seen, the three P-M spectra for several wave heights and the JONSWAP spectra also with different wave heights is shown in Fig. 3(b). Each wave spectrum is comprised dissimilar to the significant wave height, $H_{s}=5 \mathrm{~m}, 10 \mathrm{~m}, 15 \mathrm{~m}$ and different wave zero up-crossing period, $T_{z}=7.94 \mathrm{sec}, 11.23 \mathrm{sec}, 13.75 \mathrm{sec}$. In other words, the variance of spectrum frequency in either the P-M or JONSWAP spectrum is defined by using its significant wave height, $H_{s}$.

Both spectra results in Fig. 3 illustrate the number of the frequency spectrum $\left(\mathrm{m}^{2} / \mathrm{Hz}\right)$ opposed to a number of wave frequency $(\mathrm{Hz})$. It also can be described as the number of 
wave energy $\left(\mathrm{m}^{2} / \mathrm{Hz}\right)$ or energy spectrum density $\left(\sigma^{2} / \mathrm{Hz}\right)$ against a wave height $(H)$. These graphs represent a low number of small waves at the left side of the spectrum and a low number of very large waves at the right side of the spectrum. In other words, the wave height will increase when the direction becomes more and more to the right at $\mathrm{x}$-axis while the frequency spectrum makes an increment of wave numbers (frequency of occurrence) when the direction becomes more upward at the y-axis. On the other hand, most peak points fall in the mid-range of this spectrum, it is not only just the highest frequency spectrum is located but it denotes the greatest number of waves that occurred or where most probable heights $\left(H_{m}\right)$ would be. From here, it can be known if the mean wave height $(\bar{H})$ is closed to the most probable wave height $\left(H_{m}\right)$. The mean wave height $(\bar{H})$ is approximately equal to two-thirds (0.64) the value of $H_{s}$. In addition, the highest one-third number of waves in this spectrum is located in the right side of spectrum (almost 0.33 or $33 \%$ covered area of spectrum from the right to left side at a fixed certain point of frequency-axis) and the average height of waves in this 0.33 area is the significant wave height $\left(H_{s}\right)$ or the mean height of the highest one-third of the waves.

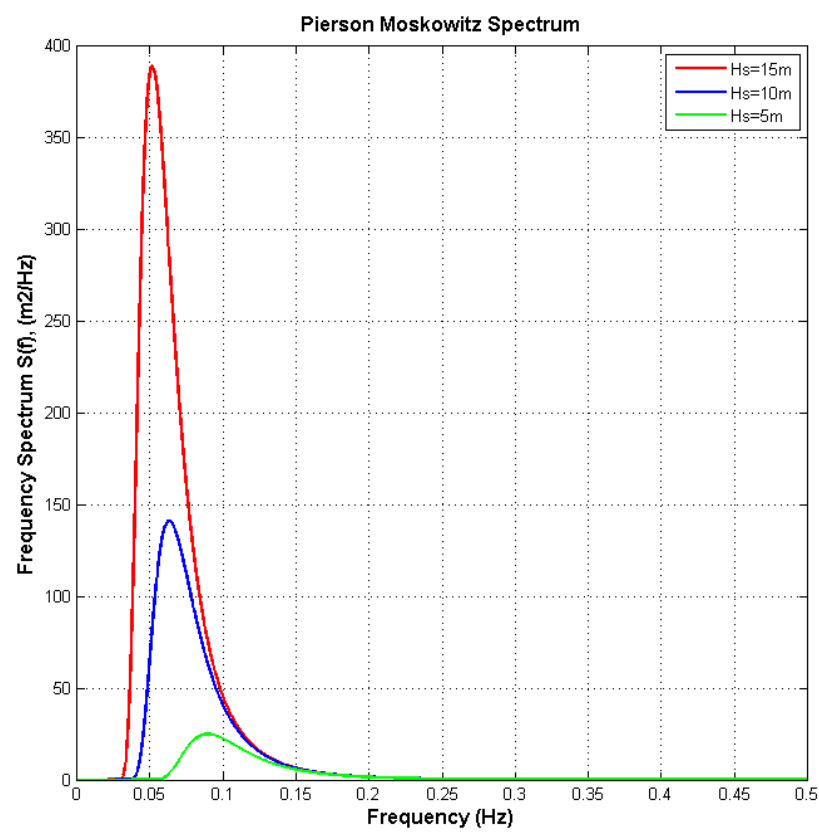

a) Pierson-Moskowitz Spectrum 


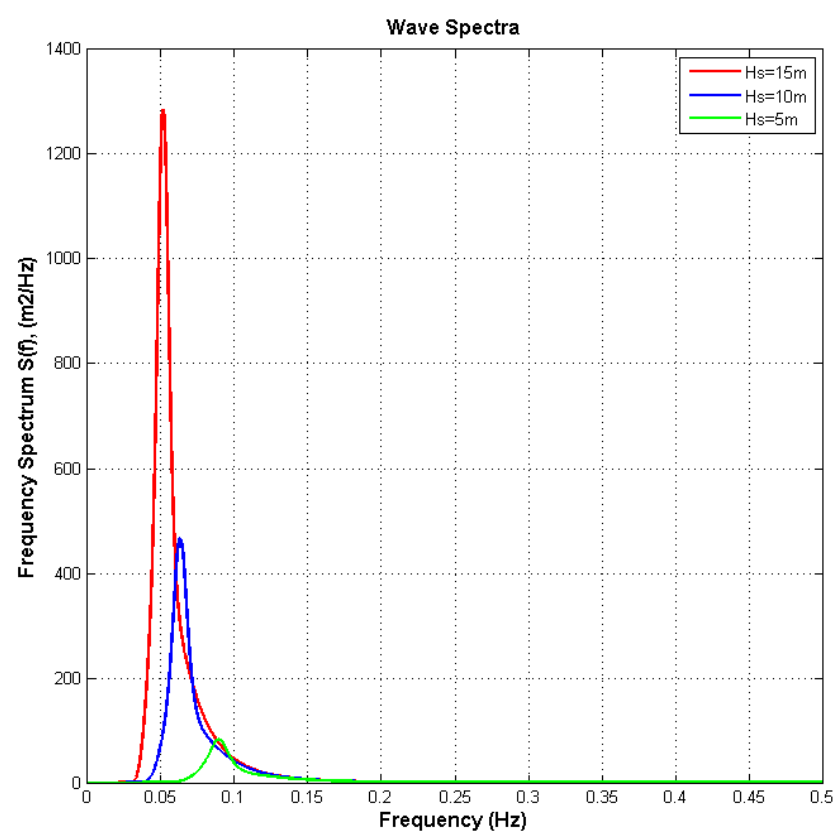

b) JONSWAP Spectrum

Fig. 3. Wave spectra for three different sea state conditions.

Fig. 4 indicates the different characteristics of two spectra with the sharp peak of the JONSWAP spectrum surpassing the PM spectrum. In this figure, the JONSWAP spectrum has a higher spectrum frequency and consequently larger significant wave heights. On the other hand, in Fig. 4 is shown the comparison between P-M wave spectrum and JONSWAP wave spectrum of sea states with the same significant wave height $\left(H_{s}\right)$ and mean zero upcrossing period $\left(T_{z}\right)$. This figure has shown the difference in maximum energy between two shapes. JONSWAP spectrum displays a more narrowed band compared to the P-M spectrum which is more likely wide band. There are more number of waves on peak frequency whereas this is more spread. In addition, the JONSWAP spectrum produces a sharp peak energy on top of the P-M spectrum. It can be called peakedness factor, where if the is wind blows at a certain speed, certain energy goes inside and tends to excite some wavelength. For more details, this can be proved by calculating the area wave spectrum ratio related to peak enhancement as below in Table 1 .

From the energy spectra analysis with random phase angle as shown in Fig. 3, the surface elevation can be reconstructed by the signal at any point and all times as mentioned in Eq. 4. Above is a sample of surface elevation time series for 128 seconds and a simulation of P-M and JONSWAP spectra are presented in Fig. 5. This graph demonstrates a simulated short time series which provides clear views of waveform compared to thousand time records. Practically, the 3.64-hour time series of surface elevation analysis would be run in this study. 


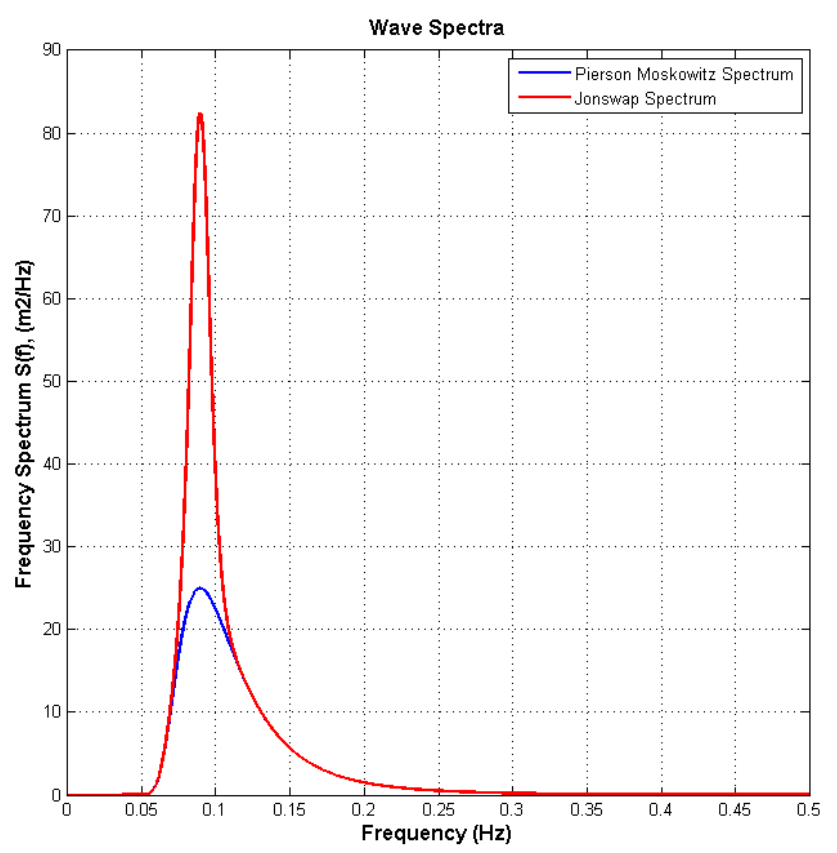

Fig. 4. Comparison of wave spectra for $H_{s}=15 \mathrm{~m}$ and $T_{z}=13.75 \mathrm{sec}$.

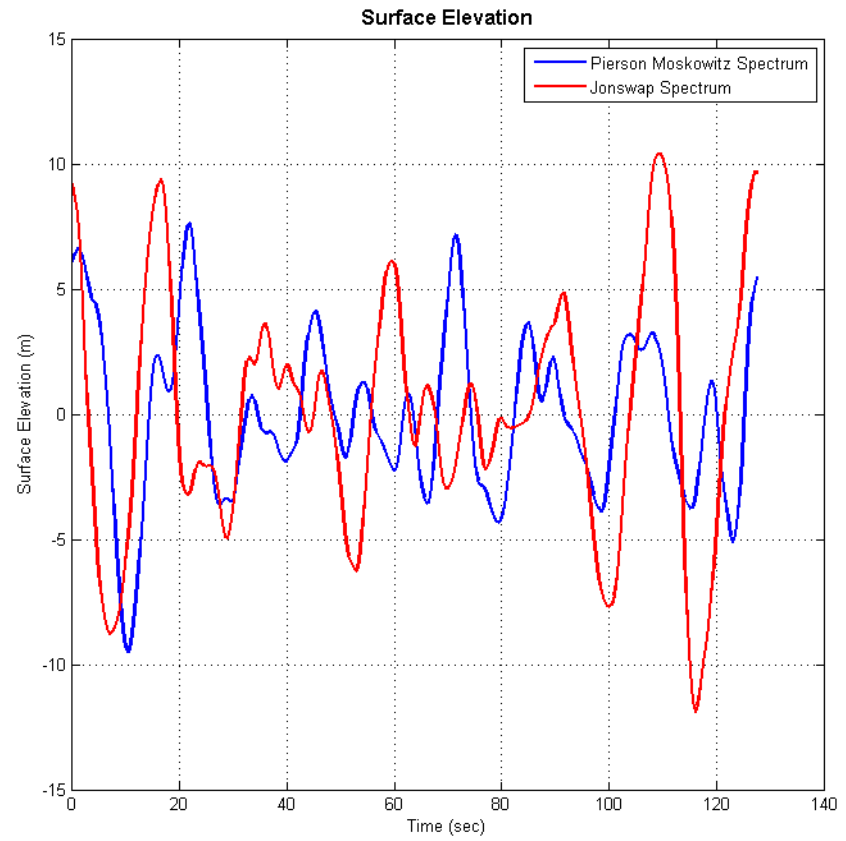

Fig. 5. A sample of P-M spectrum (blue) and JONSWAP spectrum (red) simulated surface elevation with $H_{s}=15 \mathrm{~m}, \mathrm{Tz}=13.75 \mathrm{sec}$ and $\mathrm{T}=128 \mathrm{sec}$.

As shown in Fig. 5, shown the significant wave height values appeared well correlated with graphic estimates of wave height from Fig. 4. The surface elevation between two different spectra presented that JONSWAP spectrum is much higher than P-M spectrum. The reason is the role played by spectrum frequency the JONSWAP spectrum shifted to become a higher peak period. It means the JONSWAP can produce realistic spectra and 
more flexibility due to its own five-parameter compared to two-parameter, which has that has a certain limitations in describing fully developing sea.

The final result is a demonstration of the probability distributions of the extreme values of surface elevation for a 100-year prediction that is graphically plotted to Gumbel scale. The results based on 100000 simulated extreme response records for durations $\mathrm{T}=13107$ seconds $(\approx 3.64$ hours $)$ is shown in Fig. 6 .

Based on Eq. (7), the number of 13107 second intervals during a 100 year period is $N_{100}$ $=100 * 365 * 86400 / 13104=240659.340$. Therefore, the short-term probability distribution of the extreme responses during time interval $T$ for the sea state represented by $H_{s}$ and $T_{z}$ is $4.155 \mathrm{e}^{-4}$ of percentage exceeding. The extreme value of surface elevation for each significant wave height can be referred in Table 2 .

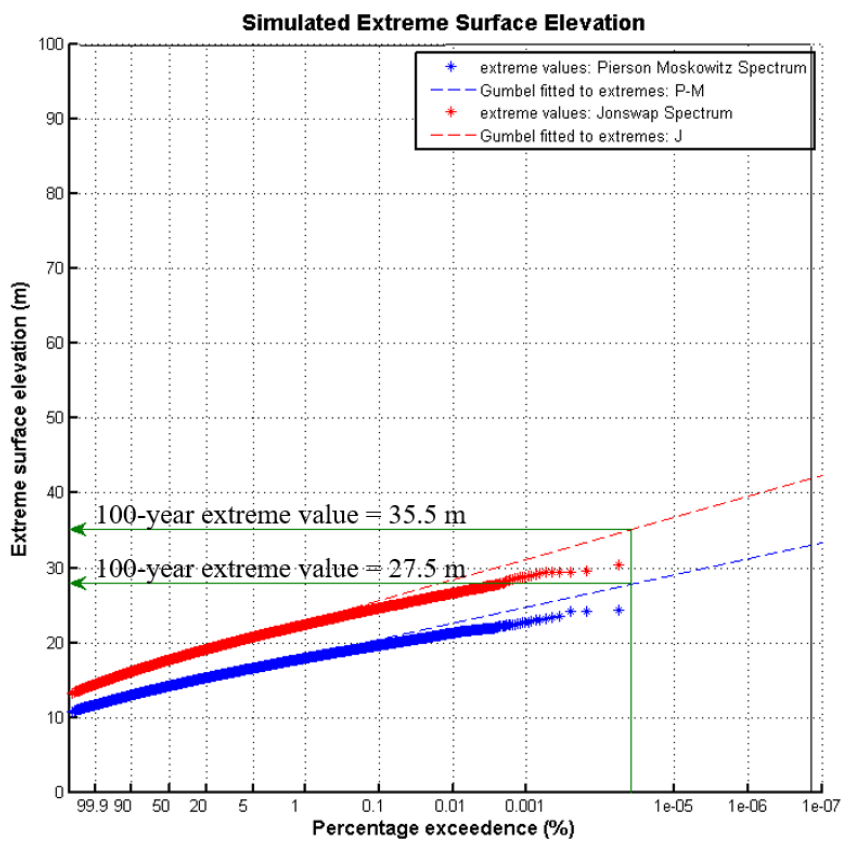

Fig. 6. Probability distribution of extreme values of surface elevation: $H_{S}=15 \mathrm{~m}, T_{z}=13.75 \mathrm{sec}$, number of response records $=100000, \mathrm{~T}=3.64$ hours.

\subsection{Verification ratio two spectra}

Both wave spectra improved that rising the spectrum frequency or energy density spectra as an increase the significant wave height. Each spectrum frequency and peak enhancement ratio are tabulated in Table 1. From that table, the spectrum frequency value experienced an increment rate proportional to the increasing wave height. However, the ratio value of peak enhancement produces the same value of 3.3 for each wave height. It denotes the ratio between JONSWAP with respect to P-M spectrum has the same value of peak enhancement that is being used in JONSWAP spectrum and achieves a good agreement in this study. In these cases, Figs. 3(a) and 3(b) show the comparison of the specified spectrum frequency between P-M spectrum and JONSWAP spectrum for the three different wave heights of $H_{s}$ $=5 \mathrm{~m}, 10 \mathrm{~m}, 15 \mathrm{~m}$, respectively. 
Table 1. Calculated spectrum frequency for the P-M and JONSWAP spectrum for various significant wave height.

\begin{tabular}{|c|c|c|c|}
\hline \multirow{2}{*}{$H_{s}(\mathbf{m})$} & \multicolumn{2}{|c|}{$S_{\omega}\left(f_{i}\right),\left(\mathrm{m}^{2} / \mathrm{Hz}\right)$} & \multirow{2}{*}{$\eta_{p}=\frac{\text { JONSWAP }_{\max }}{\mathrm{PM}_{\max }}$} \\
\hline & P-M & JONSWAP & \\
\hline 5 & 25 & 84 & 3.3 \\
\hline 10 & 135 & 445 & 3.3 \\
\hline 15 & 390 & 1290 & 3.3 \\
\hline
\end{tabular}

The P-M spectrum and JONSWAP spectrum of the extreme value surface elevation are recorded in Table 2. Each extreme value surface elevation makes an increment rate proportional to the increased value of three successive wave height. It indicates the ratio of JONSWAP over P-M spectrum delivered a steady increment value. The goodness-of-fit compares the predicted value from the extreme value surface elevation both spectra as the contribution of the firm sequence becomes more consistent with the ratio of surface elevation for each wave height result. Figs. 6 displayed how the short-term probability distribution extreme values of surface elevation was calculated at the wave height, $H_{s}=15$ $\mathrm{m}$ and wave period, $T_{z}=13.75 \mathrm{sec}$.

Table 2. Calculated extreme value surface elevation between two spectra; the P-M spectrum and JONSWAP spectrum for 100-year among design wave heights.

\begin{tabular}{|c|c|c|c|}
\hline \multirow[t]{2}{*}{$H_{s}(\mathrm{~m})$} & \multicolumn{2}{|c|}{$\begin{array}{c}\text { 100-year Wave Height } \\
\text { (m) }\end{array}$} & \multirow{2}{*}{ Ratio $=\frac{\text { JONSWAP 100-year EV of } S_{\text {int }}(f)}{\text { PM 100-year EV of } S_{\text {min }}(f)}$} \\
\hline & P-M & JONSWAP & \\
\hline 5 & 9.3 & 11.8 & 1.2688 \\
\hline 10 & 18.5 & 23.5 & 1.2702 \\
\hline 15 & 27.5 & 35.5 & 1.2909 \\
\hline
\end{tabular}

\section{Conclusion}

The time-domain analysis for the dynamic analysis of offshore structure represents more realistic random phenomena, but it takes a lot of computational time. Also, depending on the size of record, the change of time step affects the results of statistical analysis. Thus, it is important to choose the length of time record and the proper time step. The use of statistical method has been applied to estimate the spectrum frequency or energy spectrum, and how the wave energy spreads over the range of frequency and direction into the extreme value surface elevation that is located in the North Sea. The theoretical background has been addressed as case study by analysing a simple mathematical formulation, Airy's wave theory for describing waves and the height of the corresponding wave $\left(H_{s}=5 \mathrm{~m}, 10\right.$ $\mathrm{m}$ and $15 \mathrm{~m}$ ) with difference spectra. It can be concluded that by theoretical mathematical formulation of, the simulation of wave loading over mean water level, the developed program, is able to reproduce results from the mathematical modelling with satisfactory accuracy.

To sum up, fetch is a major factor in the propagation of waves and it is a major oversimplification in calculating important parameters such as wave height, wave period, frequency and spectral density. By comparing the results delivered by the MATLAB program with the previous studies and by comparing the two models, it can be verified that this program provides a reasonable representation of the JONSWAP and PM wave spectrum models. The effect of fetching parameters in JONSWAP spectrum would give a 
higher embedded energy spectrum compared to the P-M spectrum. The number of different parameter usage between the P-M spectrum models with two-parameter spectrum and the JONSWAP spectrum with five parameters also make a difference in the final results. This is the reason why the P-M spectrum gives a bit more freedom to reproduce realistic spectra of developing sea. Otherwise, the existing JONSWAP spectrum offers more flexibility and can produce more realistic spectra compared to P-M spectrum.

In the short-term probability distribution description of the wave characteristics, the three significant wave heights have been determined with a Gumbel distribution. Two different wave frequency spectra, the P-M spectrum and JONSWAP spectrum, have been selected to see the effect of estimating extreme surface elevation wave height for 100-year period. The results have been recorded in this study.

This work was supported by the Universiti Teknologi Malaysia [grant no: Q.K130000.2540.09H39 / Q.K130000.2540.09H42] and the Ministry of Higher Education (Malaysia) [grant no: R.K130000.7940.4F584 / R.K130000.7840.4F583].

\section{References}

1. Y. Goda, Random Seas and Design of Maritime Structures, ( $3^{\text {rd }}$ Ed. 33, 1985).

2. X. G. Larsén, C. Kalogeri, G. Galanis, G. Kallos, A Statistical Methodology for The Estimation of Extreme Wave Conditions for Offshore Renewable Applications, Renewable Energy, Elsevier, 80, pp. 205-218 (2015)

3. T. Sarpkaya, M. Isaacson, Mechanics of Wave Forces on Offshore Structures, New York: Van Nostrand Reinhold Co, (1981).

4. Chapter 4 The Monte Carlo Method. Mathematics in Science and Engineering. G. Arthur Mihram, Elsevier. 92, 181-208, (1972)

5. J. J. Jensen, Extreme Value Predictions uing Monte Carlo Simulations with Artificially Increased Load Spectrum, Probab. Eng. Mech., 26, 2, pp. 399-404, (2011)

6. M. K. Abu Husain, G. Najafian, An Efficient Monte Carlo Simulation Technique for Derivation of the Probability Distribution of the Extreme Values of Offshore Structural Response. ASME 2010 29th International Conference on Ocean, Offshore and Arctic Engineering, 2, pp. 369-375 (2010)

7. M. K. Abu Husain, N. I. Mohd Zaki, M. B. Johari, G. Najafian, Extreme Response Prediction for Fixed Offshore Structures by Monte Carlo Time Simulation Technique. ASME 2016 35th International Conference on Ocean, Offshore and Arctic Engineering, 3, pp. V003T02A037 (2016)

8. C. Stuart, An Introduction to Statistical Modeling of Extreme Values. London: Springer, (2001)

9. A. D. D. Craik, The Origins of Water Wave Theory, Annual Review of Fluid Mechanics. 36, pp. 1-28 (2004).

10. S, Richard, Dynamics of Marine Sands: A Manual For Practical Applications. London: Telford, pp. 90-146, (1997)

11. J. Witz, G. Lyons, M. H. Patel, D. Brown, Advanced Offshore Engineering. Offshore Engineering Handbook Series. 1st ed. Bentham Press, London, UK, pp. 5.2-5.10. (1994)

12. M., Onorato, Coauthors, Statistical Properties of Mechanically Generated Surface Gravity Waves: A Laboratory Experiment in a Three-Dimensional Wave Basin. J. Fluid Mech., 637, pp. 235-257 (2009a) 
13. S. K. Chakrabarti, Handbook of Offshore Engineering I, (2005)

14. CEM, 'Coastal Engineering Manual', Coastal Engineering Research Center, Dept. of Army Corps of Engineers, US, Chapter I, Part 2. (2006)

15. L. E. Borgman "Ocean Wave Simulation for Engineering Design," Journal of the Waterways and Harbors Division, ASCE, 95, WW4, Proc. pp. 557-583. Nov (1969)

16. C. Guedes Soares, M. C. Nolasco, Spectra Modeling of Sea States with Multiple Wave Systems, J. Offshore Mech. Arct. Eng., 114, pp. 278-284 (1992)

17. J. Westphalen, Extreme Wave Loading on Offshore Wave Energy Devices using CFD, University of Plymouth, Page. 241, (2011)

18. A. G. Davenport, The Relationship of Reliability to Wind Loading, Journal of Wind Engineering and Industrial Aerodynamics, 1-3, 13, pp. 3-27 (1983)

19. Jr. W. J. Pierson, L. Moskowitz, A Proposed Spectral Form for Fully Developed Wind Seas Based on The Similarity Theory of S. A. Kitaigorodskii, J. Geophys. Res., 69, 24, pp. 5181-5190 (1964)

20. K. Hasselmann, T. P. Barnett, E. Bouws, H. Carlson, D. E. Cartwright, K. Enke, 'Measurements of Wind-Wave Growth and Swell Decay During the Joint North Sea Wave Project (JONSWAP)', Deutsche Hydr Zeit, A 8, 12, pp. 95 (1973)

21. O. M. Faltinsen, Sea Loads on Ships and Offshore Structures. (Cambridge: Cambridge University Press, 1990)

22. J. J. Jensen, I. M. V. Andersen, S. Seng, Stochastic Procedures for Extreme Wave Induced Responses in Flexible Ships, International Journal of Naval Architecture and Ocean Engineering, 6, 4, pp. 1148-1159 (2014)

23. S. K. Haver, Prediction of Characteristic Response for Design Purpose. Statoil. (2013)

24. Y. Wang, H. Mallahzadeh, M. K. Abu Husain, N. I. Mohd Zaki, G. Najafian, Probabilistic Modelling of Extreme Offshore Structural Response due to Random Wave Loading. ASME 2013 32nd International Conference on Ocean, Offshore and Arctic Engineering, 2B, pp. V02BT02A007, (2013) 